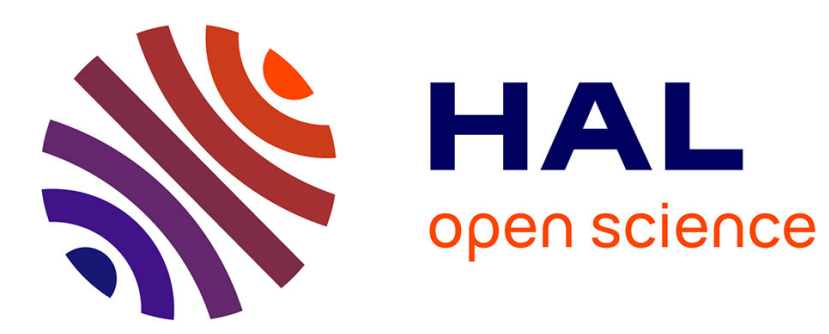

\title{
Complete stability analysis of a control law for walking robots with non-permanent contacts \\ Sophie Chareyron, Pierre-Brice Wieber
}

\section{To cite this version:}

Sophie Chareyron, Pierre-Brice Wieber. Complete stability analysis of a control law for walking robots with non-permanent contacts. International Conference on Climbing and Walking Robots, 2005, London, United Kingdom. inria-00390419

\section{HAL Id: inria-00390419 \\ https://hal.inria.fr/inria-00390419}

Submitted on 2 Jun 2009

HAL is a multi-disciplinary open access archive for the deposit and dissemination of scientific research documents, whether they are published or not. The documents may come from teaching and research institutions in France or abroad, or from public or private research centers.
L'archive ouverte pluridisciplinaire HAL, est destinée au dépôt et à la diffusion de documents scientifiques de niveau recherche, publiés ou non, émanant des établissements d'enseignement et de recherche français ou étrangers, des laboratoires publics ou privés. 


\title{
Complete stability analysis of a control law for walking robots with non-permanent contacts
}

\author{
Sophie Chareyron ${ }^{\star} 1$ and Pierre-Brice Wieber ${ }^{1}$ \\ INRIA Rhône-Alpes, ZIRST-655 avenue de l'Europe-Montbonnot-38335 Saint \\ Ismier Cedex \\ sophie.chareyron@inrialpes.fr,Pierre-Brice.Wieber@inrialpes.fr
}

\section{Introduction}

One of the main specificities of walking robots is their non-permanent contact with the ground which impairs their stability. The only stability analyses of control laws that have been proposed so far for walking robots have been distinctly focusing on each contact phases, with the strong assumption that these phases are never perturbated $[11,13]$. In this study we aim at analysing the stability of a regulation of the position of a walking robot without any assumption on the state of these contacts. In order to do so, we proposed in [2] to work in the framework of nonsmooth dynamical systems, what provides a general formulation of the dynamics that does not depend on the contact state. Classical stability theorems cannot be applied to this framework, so we needed to derive in [2] a Lyapunov stability theorem and a Lagrange Dirichlet theorem specifically for Lagrangian dynamical systems with non-permanent contacts. Based on these theorems, we will prove here the stability of a simple control action that realizes the regulation of the position and contact forces of a walking robot.

\section{Position and force regulation law for walking robots}

We first present in section 2.1 the model used to describe walking machines with non-permanent contacts. In sections 2.2 and 2.3 we will see that walking machines can be seen as underactuated systems, and thus a control law such as the one studied in [3] for a robotic manipulator cannot be used directly. Therefore, we propose in section 2.4 a control law designed in a similar way but adapted to the regulation of the position and contact forces of a walking robot.

\footnotetext{
* This work was supported by the European project SICONOS IST2001-37172, and was (partially) done in the framework of the HYCON Network of Excellence, contract number FP6-IST-511368*
} 


\subsection{Walking machines with non-permanent contacts}

With $n$ the number of degrees of freedom of the walking robot, let us consider a time-variation of generalized coordinates $\boldsymbol{q}: \mathbb{R} \rightarrow \mathbb{R}^{n}$ and the related velocity $\dot{\boldsymbol{q}}: \mathbb{R} \rightarrow \mathbb{R}^{n}:$

$$
\forall t, t_{0} \in \mathbb{R}, \boldsymbol{q}(t)=\boldsymbol{q}\left(t_{0}\right)+\int_{t_{0}}^{t} \dot{\boldsymbol{q}}(\tau) d \tau
$$

We assume that the interaction between the feet of the walking machine and the ground is modelled by non-penetrating rigid bodies, which implies that the feet cannot penetrate or stick on the ground. That can be described by a set of unilateral constraints on the position of the system

$$
\boldsymbol{\varphi}(\boldsymbol{q}) \geq 0, \boldsymbol{\varphi}: \mathbb{R}^{n} \rightarrow \mathbb{R}^{m} .
$$

The dynamics of walking robots can be described in this case by a classical Lagrange differential equation:

$$
\boldsymbol{M}(\boldsymbol{q}) \frac{d \dot{\boldsymbol{q}}}{d t}+\boldsymbol{N}(\boldsymbol{q}, \dot{\boldsymbol{q}}) \dot{\boldsymbol{q}}+\boldsymbol{g}(\boldsymbol{q})=\boldsymbol{u}+\boldsymbol{r}+\boldsymbol{f}
$$

with $\boldsymbol{M}(\boldsymbol{q})$ the inertia matrix, $\boldsymbol{N}(\boldsymbol{q}, \dot{\boldsymbol{q}}) \dot{\boldsymbol{q}}$ the corresponding nonlinear effects, $\boldsymbol{g}(\boldsymbol{q})$ the gravity forces, $\boldsymbol{u}$ the control action, $\boldsymbol{r}$ and $\boldsymbol{f}$ the normal and tangential contact forces (also referred as the reaction and friction forces). In the following, we are not going to precise here the model of these contact forces, we will only use the dissipativity property of friction: $\boldsymbol{f}^{T} \dot{\boldsymbol{q}} \leq 0$.

\subsection{Walking machines as underactuated systems}

First note that the vector of generalized coordinates $\boldsymbol{q}$ of a walking robot can be shown [14] to have the following structure,

$$
\boldsymbol{q}=\left[\begin{array}{l}
\boldsymbol{q}_{1} \\
\boldsymbol{q}_{2}
\end{array}\right]
$$

with $\boldsymbol{q}_{1}$ a vector gathering the position of the robot articulations and $\boldsymbol{q}_{2}$ a vector describing the position and orientation of one solid of the robot with respect to the environment.

Since the actuators of the robot produce a torque $\boldsymbol{\tau}$ that acts only on the positions of its articulations, the actuation $\boldsymbol{u}$ can be shown therefore [15] to have the following structure

$$
\boldsymbol{u}=\left[\begin{array}{l}
\boldsymbol{\tau} \\
\mathbf{0}
\end{array}\right]
$$

\subsection{Contact forces at equilibrium positions}

With the actuation (2), the robot dynamics (1) is then given by

$$
\boldsymbol{M}(\boldsymbol{q}) \frac{d \dot{\boldsymbol{q}}}{d t}+\boldsymbol{N}(\boldsymbol{q}, \dot{\boldsymbol{q}}) \dot{\boldsymbol{q}}+\boldsymbol{g}(\boldsymbol{q})=\left[\begin{array}{l}
\boldsymbol{\tau} \\
\mathbf{0}
\end{array}\right]+\boldsymbol{r}+\boldsymbol{f}
$$


and at equilibrium points, when $\dot{\boldsymbol{q}}=0$ and $\frac{d \dot{\boldsymbol{q}}}{d t}=0$, it reduces to

$$
\boldsymbol{r}+\boldsymbol{f}=\boldsymbol{g}(\boldsymbol{q})-\left[\begin{array}{c}
\boldsymbol{\tau} \\
0
\end{array}\right] \text { or }\left[\begin{array}{l}
\boldsymbol{r}_{1}+\boldsymbol{f}_{1} \\
\boldsymbol{r}_{2}+\boldsymbol{f}_{2}
\end{array}\right]=\left[\begin{array}{c}
\boldsymbol{g}_{1}(\boldsymbol{q})-\boldsymbol{\tau} \\
\boldsymbol{g}_{2}(\boldsymbol{q})
\end{array}\right]
$$

Relation (3) shows an equilibrium condition between the three external forces: the contact forces (normal and tangential), the gravity forces and the actuation. Due to the structure of the actuation (2), $\boldsymbol{\tau}$ appears only in the upper part of this equilibrium condition. So the lower part of this equilibrium condition (3) relates only the reaction forces and the gravity forces independently of the actuation:

$$
\boldsymbol{r}_{2}+\boldsymbol{f}_{2}=\boldsymbol{g}_{2}(\boldsymbol{q})
$$

This equilibrium condition can be related to more usual equilibrium conditions of mechanical systems $[7,15]$. Indeed, the dynamics (1) can be shown to have a structure matching the one of the equilibrium condition (3), and as proved in [14], the second part of this dynamics corresponds to the Newton and Euler equations of the robot. In this way, the second part of equation (3) can be shown to provide an equilibrium condition between the position of the center of mass and the positions of the contacts with the ground, see [15] for more details.

The upper part of the equilibrium condition (3) relates the part $\boldsymbol{r}_{1}+\boldsymbol{f}_{1}$ of the contact forces to the gravity forces and the the actuation

$$
\boldsymbol{r}_{1}+\boldsymbol{f}_{1}=\boldsymbol{g}_{1}(\boldsymbol{q})-\boldsymbol{\tau} .
$$

Though it seems that the contact forces $\boldsymbol{r}_{1}+\boldsymbol{f}_{1}$ can be set to any value through the parameter $\tau$, the inner structure of the friction and reaction forces (not precised here) entails that the parts $\boldsymbol{r}_{1}+\boldsymbol{f}_{1}$ and $\boldsymbol{r}_{2}+\boldsymbol{f}_{2}$ of the reaction forces are in fact completely intertwined, thus $\boldsymbol{r}_{1}+\boldsymbol{f}_{1}$ also strongly depends on the equilibrium condition (4).

\subsection{A control law through potential shaping}

We want to realize a regulation of the position and contact forces of a walking robot to some desired position $\boldsymbol{q}_{d}$ and desired contact forces $\boldsymbol{r}_{d}+\boldsymbol{f}_{d}$. The torque $\boldsymbol{\tau}$ has to be designed to compensate the part $\boldsymbol{g}_{1}\left(\boldsymbol{q}_{d}\right)$ of the gravity forces so that we can obtain the desired reaction forces $\boldsymbol{f}_{1 d}+\boldsymbol{r}_{1 d}$ at the desired position $\boldsymbol{q}_{d}$

$$
\boldsymbol{\tau}\left(\boldsymbol{q}_{d}\right)=\boldsymbol{g}_{1}\left(\boldsymbol{q}_{d}\right)-\boldsymbol{r}_{1 d}-\boldsymbol{f}_{1 d}
$$

In order to do so, we'll consider a control law designed through potential shaping, and more precisely, following [12], we choose the following potential function

$$
\tilde{\mathrm{P}}\left(\boldsymbol{q}_{1}\right)=\frac{1}{2}\left(\boldsymbol{q}_{1}-\boldsymbol{q}_{1 d}\right)^{T} \boldsymbol{W}\left(\boldsymbol{q}_{1}-\boldsymbol{q}_{1 d}\right)+\left(\boldsymbol{r}_{1 d}+\boldsymbol{f}_{1 d}-\boldsymbol{g}_{1}\left(\boldsymbol{q}_{d}\right)\right)^{T}\left(\boldsymbol{q}_{1}-\boldsymbol{q}_{1 d}\right),
$$

with $\boldsymbol{W}$ a symmetric positive definite matrix and $\boldsymbol{q}_{1 d}$ the desired position of the robot articulations. The derivative of this potential function is 


$$
\frac{d \tilde{\mathrm{P}}}{d \boldsymbol{q}_{1}}\left(\boldsymbol{q}_{1}\right)=\boldsymbol{W}\left(\boldsymbol{q}_{1}-\boldsymbol{q}_{1 d}\right)+\boldsymbol{r}_{1 d}+\boldsymbol{f}_{1 d}-\boldsymbol{g}_{1}\left(\boldsymbol{q}_{d}\right),
$$

to which we add a dissipative term $\boldsymbol{T} \dot{\boldsymbol{q}}_{1}$, with $\boldsymbol{T}$ a positive definite matrix, in order to obtain the following Proportionnal Derivative control law with precompensation of the gravity and desired contact forces:

$$
\boldsymbol{\tau}=-\boldsymbol{W}\left(\boldsymbol{q}_{1}-\boldsymbol{q}_{1 d}\right)+\boldsymbol{g}_{1}\left(\boldsymbol{q}_{d}\right)-\boldsymbol{r}_{1 d}-\boldsymbol{f}_{1 d}-\boldsymbol{T} \dot{\boldsymbol{q}}_{1} .
$$

\section{Nonsmooth dynamical systems}

In order to prove the stability of the control law (6) without any assumption on the state of the contacts, we will use in section 3.3 some stability results for nonsmooth Lagrangian dynamical systems as proposed in [2]. The sections 3.1 and 3.2 aim therefore at presenting very briefly the framework of nonsmooth Lagrangian dynamical systems and its mathematical tools from convex analysis and measure theory that are unusual in control theory. For a more complete presentation, refer to [2].

\subsection{Some geometry for systems with non-permanent contacts}

We have seen in section 2.1 that the non-penetration of perfectly rigid bodies can be expressed as a constraint on the robot position, a constraint that will take the form of a closed set

$$
\Phi=\left\{\boldsymbol{q} \in \mathbb{R}^{n} \mid \boldsymbol{\varphi}(\boldsymbol{q}) \geq 0\right\} .
$$

in which the generalized coordinates $\boldsymbol{q}$ are bound to stay:

$$
\forall t \in \mathbb{R}, \boldsymbol{q}(t) \in \Phi .
$$

When $\boldsymbol{q}$ is in the interior of the domain $\Phi$, there is no interaction between the walking robot and its environment. On the other hand, when the system lies on the boundary of $\Phi$, the robot and its environment interact, what generates contacts forces $\boldsymbol{r}+\boldsymbol{f}$. Concerning the normal forces, this fact can be described trough the inclusion

$$
-\boldsymbol{r} \in \mathcal{N}(\boldsymbol{q})
$$

involving the normal cone $\mathcal{N}(\boldsymbol{q})$ of $\Phi$ at $\boldsymbol{q}$ as defined in [4] and as illustrated in figure 1.

Now, we can observe that when the system reaches the boundary of $\Phi$ with a velocity $\dot{\boldsymbol{q}}^{-}$directed outside of this domain, it won't be able to continue its movement with a velocity $\dot{\boldsymbol{q}}^{+}=\dot{\boldsymbol{q}}^{-}$and still stay in $\Phi$ (Fig. 1). A discontinuity of the velocity will have to occur then, corresponding to an impact between contacting rigid bodies. This can be described by the fact that the velocity $\dot{\boldsymbol{q}}^{+}$has to belong any time to the tangent cone $\mathcal{T}(\boldsymbol{q})$ of $\Phi$ at $\boldsymbol{q}$ as defined in [4] and as illustrated in figure 1 . Note that the velocity after this impact $\dot{\boldsymbol{q}}^{+}$can be related to the velocity before the impact $\dot{\boldsymbol{q}}^{-}$by modelling this impulsive 
behavior through a contact law. We are not going to precise here this contact law, we will only use the fact that the impact is a dissipative phenomenom, which implies that the kinetic energy $K(\boldsymbol{q}, \dot{\boldsymbol{q}})$ satisfies

$$
K\left(\boldsymbol{q}, \dot{\boldsymbol{q}}^{+}\right) \leq K\left(\boldsymbol{q}, \dot{\boldsymbol{q}}^{-}\right) .
$$

For a more in-depth presentation of these concepts and equations which may have subtle implications, the interested reader should definitely refer to [9] or $[2,3]$.

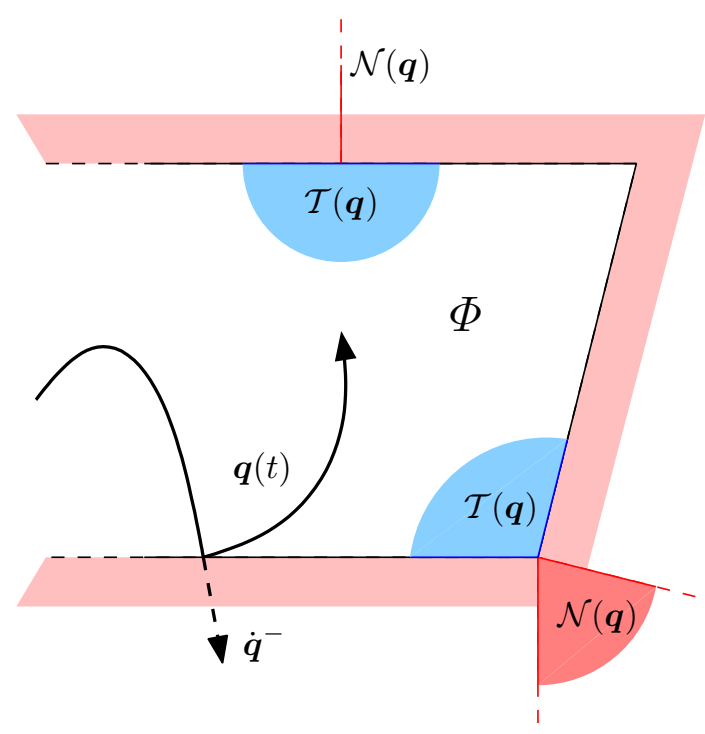

Fig. 1. Examples of tangent cones $\mathcal{T}(\boldsymbol{q})$ and normal cones $\mathcal{N}(\boldsymbol{q})$ on the boundary of the domain $\Phi$, and example of a trajectory $\boldsymbol{q}(t) \in \Phi$ that reaches this boundary with a velocity $\dot{\boldsymbol{q}}^{-} \notin \mathcal{T}(\boldsymbol{q})$.

\subsection{Nonsmooth Lagrangian dynamical systems}

Classically, solutions to the dynamics of Lagrangian systems such as (1) lead to smooth motions with locally absolutely continuous velocities. But we have seen in section 3.1 that discontinuities of the velocities may occur when the coordinates of such systems are constrained to stay inside closed sets. These classical differential equations must therefore be turned into measure differential equations [9]

$$
\boldsymbol{M}(\boldsymbol{q}) d \dot{\boldsymbol{q}}+\boldsymbol{N}(\boldsymbol{q}, \dot{\boldsymbol{q}}) \dot{\boldsymbol{q}} d t+\boldsymbol{g}(\boldsymbol{q}) d t=\boldsymbol{u} d t+d \boldsymbol{r}+\boldsymbol{f} d t,
$$

where the reaction forces are now represented by an abstract measure $d \boldsymbol{r}$ which may not be Lebesgues-integrable. This way, the measure acceleration $d \dot{\boldsymbol{q}}$ may not be Lebesgues-integrable either so that the velocity may not be locally 
absolutely continuous anymore but only with locally bounded variation, $\dot{\boldsymbol{q}} \in$ $\operatorname{lbv}\left(\mathbb{R}, \mathbb{R}^{n}\right)[9]$. Functions with locally bounded variation have left and right limits at every instant, and we have for every compact subinterval $[\sigma, \tau] \subset \mathbb{R}$

$$
\int_{[\sigma, \tau]} d \dot{\boldsymbol{q}}=\dot{\boldsymbol{q}}^{+}(\tau)-\dot{\boldsymbol{q}}^{-}(\sigma)
$$

A function $f$ has a locally bounded variation on $\mathbb{R}$ if its variation on any compact interval $\left[t_{0}, t_{n}\right]$ is finite:

$$
\operatorname{Var}\left(f ;\left[t_{0}, t_{n}\right]\right)=\sup _{t_{0} \leq \ldots \leq t_{n}} \sum_{i=1}^{n}\left\|f\left(t_{i}\right)-f\left(t_{i-1}\right)\right\|<+\infty .
$$

Rather than for this definition, it is for their properties that functions with bounded variations are useful. Notably, functions with locally bounded variation can be decomposed into the sum of a continuous function and a countable set of discontinuous step functions [8]. In specific cases, as when the definition of the dynamics (9) is piecewise analytic, its solutions can be shown to be piecewise continuous with possibly infinitely (countably) many discontinuities [1]. In this case, it is possible to focus distinctly on each continuous piece and each discontinuity as in the framework of hybrid systems [6]. But this is usually done through an ordering of the discontinuities strictly increasing with time, what is problematic when having to go through accumulations of impacts. The framework of nonsmooth analysis appears therefore as more appropriate for the analysis of impacting systems, even though the calculus rules for functions with bounded variation require some care.

\subsection{Some Lyapunov stability theory}

The Lyapunov stability theory is usually presented for dynamical systems with states that vary continuously with time [5], [17], Fillipov systems for example [10], but we have seen that in the case of nonsmooth mechanics, the velocity and thus the state may present discontinuities. Lyapunov stability theory is hopefully not strictly bound to continuity properties, and some results can still be derived for discontinuous dynamical systems both in the usual framework of hybrid systems [16] and in the framework of nonsmooth analysis [2].

In the following we will prefer the latter for the reasons mentioned in section 3.2, for which we proposed in [2] the following corollary of a LagrangeDirichlet theorem:

Corollary 1. Consider a nonsmooth Lagrangian dynamical system experiencing external forces composed of normal contact forces and other Lebesguesintegrable forces $\mathcal{F}$. If these Lebesgues-integrable forces derive from a coercive $C^{1}$ potential function $P(\boldsymbol{q})$ with a dissipative term $\boldsymbol{h}$ :

$$
\mathcal{F}=-\frac{d P}{d \boldsymbol{q}}(\boldsymbol{q})+\boldsymbol{h}, \text { with } \dot{\boldsymbol{q}}^{T} \boldsymbol{h} \leq 0,
$$

then the set $\mathcal{S}=\{\operatorname{Arg} \min P(\boldsymbol{q})\} \times\{\mathbf{0}\}$ is Lyapunov stable. 
In our case, the Lebesgues-integrable forces $\mathcal{F}$ acting on the dynamics (9) are composed of the gravity forces, the actuation, and the friction forces,

$$
\mathcal{F}=-\boldsymbol{g}(\boldsymbol{q})+\boldsymbol{u}+\boldsymbol{f}
$$

With $G(\boldsymbol{q})$ the potential of the gravity forces, if we replace the control law $\boldsymbol{u}$ by its expresion (6), $\mathcal{F}$ can be expressed as the derivative of potential functions plus dissipative terms

$$
\mathcal{F}=-\frac{d G}{d \boldsymbol{q}}(\boldsymbol{q})-\frac{d \tilde{\mathrm{P}}}{d \boldsymbol{q}}(\boldsymbol{q})-\left[\begin{array}{c}
\boldsymbol{T} \\
\mathbf{0}
\end{array}\right] \dot{\boldsymbol{q}}_{1}+\boldsymbol{f}
$$

deriving therefore from the potential $P(\boldsymbol{q})=G(\boldsymbol{q})+\tilde{\mathrm{P}}\left(\boldsymbol{q}_{1}\right)$, and we can conclude through corollary 1 on the stability of the set $\mathcal{S}=\{\operatorname{Arg} \min P(\boldsymbol{q})\} \times\{\mathbf{0}\}$ without making any assumption on the state of the contacts.

Now, as shown in $[2,3]$, this set corresponds to the equilibrium positions of the system. The control law (6) has been designed so that the desired position $\boldsymbol{q}_{d}$ is an equilibrium position. We can assume under mild conditions [3] that it is the only one and it is therefore stable.

\subsection{Why such a "simple" control law}

First of all, note that the control action (6) can't compensate the impulsive behaviors of the contacts. Now, we have seen in section 3.1 that these impulsive behaviors originating from the discontinuities of the velocity are related to the kinetic energy through relation (8). It is therefore natural to use the energy of the system for the stability analysis, leading to the Lagrange-Dirichlet theorem for nonsmooth Lagrangian dynamical systems [2] and its corollary 1 that is used here. Since the control law (6) has been designed by potential shaping, the energy of the system appears as a natural candidate for its stability analysis. For the same reason, the choice of a control law trying to compensate completely the system dynamics such as a computed torque [11, 13], appears to be not very judicious because this energy can't be used any longer for the stability analysis. Finally, it is not possible to compensate completely the external forces in the case of walking machines because of the underactuation (2) what explains the difference with the control law proposed in [3].

\section{Conclusion}

In this study we aimed at analysing the stability of a regulation of the position of a walking robot without any assumption on the state of the contacts. We thus proposed to work in the framework of nonsmooth dynamical systems since it provides a general formulation of the dynamics that does not depend on the contact state. Classical stability results cannot be applied in this framework, so we needed to derive in [2] a Lyapunov stability theorem for Lagrangian dynamical systems with non-permanent contacts. Based on this theorem, we then proved the stability of a simple control law that realizes the regulation of the position and contact forces of a walking robot. Through this study, we were thus able to propose for the first time a complete stability analysis of the regulation of the position of a walking robot. 


\section{References}

1. P. Ballard. The dynamics of discrete mechanical systems with perfect unilateral constraints. Archive for Rational Mechanics and Analysis, (154):199-274, 2000.

2. S. Chareyron and P.B. Wieber. Stabilization and regulation of nonsmooth lagrangian systems. Technical Report 5408, INRIA Rhône-Alpes, 2004.

3. S. Chareyron and P.B. Wieber. Position and force control of nonsmooth lagrangian dynamical systems without friction. In Lothar Thiele Manfred Morari, editor, Lecture Notes in Computer Science, volume 3414, page 215. SpringerVerlag GmbH, Feb 2005.

4. J.-B. Hiriart-Urruty and C. Lemaréchal. Convex Analysis and Minimization Algorithms. Springer Verlag, 1996. Two volumes - 2nd printing.

5. H.K. Khalil. Nonlinear systems. Prentice-Hall, 1996.

6. J. Lygeros, K.H. Johansson, S.N. Simic, J. Zhang, and S.S. Sastry. Dynamical properties of hybrid automata. IEEE Transactions on Automatic Control, 48:217, 2003.

7. Richard Mason, Elon Rimon, and Joel Burdick. A general stability test for multiply contacted objects in a potential field, with example applications to planar objects under gravity. IEEE Transactions on Robotics and Automation, December 1996. (submitted).

8. J.-J. Moreau. Bounded variation in time. In J.-J. Moreau, P.D. Panagiotopulos, and G. Strang, editors, Topics in Nonsmooth Mechanics, pages 1-74. Birkhäuser, 1988.

9. J.-J. Moreau. Unilateral contact and dry friction in finite freedom dynamics. In J.-J. Moreau and P.D. Panagiotopulos, editors, Nonsmooth mechanics and Applications, volume 302, pages 1-82. Springer Verlag, 1988.

10. Y. Orlov. Extended invariance principle for nonautonomous switched systems. IEEE Transactions on Automatic Control, 48(8):1448-1452, august 2003.

11. Jonghoon Park, Youngil Youm, and Wan-Kyun Chung. Control of ground interaction at the zero-moment point for dynamic control of humanoid robots. Barcelona, Spain, April 2005. Proceedings of the 2005 IEEE International Conference on Robotics and Automation.

12. D. Wang and H. McClamroch. Position and force control for constrained manipulator motion: Lyapunov's direct method. IEEE Trans. Robot. Automat. 9(3):308-313, 1993.

13. E. R. Westervelt, J. W. Grizzle, and D. E. Koditschek. Hybrid zero dynamics of planar biped walkers. IEEE Transactions on Automatic Control, 48(1):42-56, January 2003.

14. P. B. Wieber. Modélisation et commande d'un robot marcheur anthropomorphe. Phd thesis, Mines de Paris, 2000.

15. P. B. Wieber. On the stability of walking systems. In Proceedings of the International Workshop on Humanoid and Human Friendly Robotics, 2002.

16. H. Ye, A. Michel, and L. Hou. Stability theory for hybrid dynamical systems. IEEE Transactions on Automatic Control, 43(4):461-474, 1998.

17. V.I. Zubov. Methods of A.M. Lyapunov and their application. Noordhoff, 1964. 